\title{
RECUPERAÇÃO DE COBRE DE PLACAS DE CIRCUITO IMPRESSO ESGOTADAS A PARTIR DE EXTRAÇÃO COM CO2 SUPERCRÍTICO
}

\author{
D. F. SCHLEMMER ${ }^{1}$, C. O. CALGARO ${ }^{1}$, M. D. SILVA ${ }^{1}$, E. TANABE ${ }^{1}$, D. A. BERTUOL ${ }^{1}$ \\ ${ }^{1}$ Universidade Federal de Santa Maria, Departamento de Engenharia Química \\ E-mail para contato: dfschlemmer@gmail.com
}

\begin{abstract}
RESUMO - A demanda cada vez maior por equipamentos elétricos e eletrônicos trouxe a necessidade do desenvolvimento de rotas tecnológicas para o reaproveitamento ou recuperação de seus constituintes. As placas de circuito impresso (PCIs) representam uma fração significativa dessa demanda, uma vez que são constituintes essenciais dos equipamentos elétricos e eletrônicos, As PCIs são compostas por materiais cerâmicos, plásticos e metais, com destaque para o Cobre $(\mathrm{Cu})$, metal mais abundante nessas placas. Levando isso em consideração o objetivo desse trabalho foi recuperar o $\mathrm{Cu}$ (cobre) das PCIs utilizando $\mathrm{CO}_{2}$ supercrítico. O estudo compreendeu a separação das PCIs de celulares descartados; sua moagem em moinho de martelos e facas; sua caracterização e, a extração supercrítica com $\mathrm{CO}_{2}$ na presença dos cosolventes $\mathrm{H}_{2} \mathrm{SO}_{4}$ e $\mathrm{H}_{2} \mathrm{O}_{2}$. Determinou-se a presença de $34,83 \%$ em massa de $\mathrm{Cu}$ nas PCIs, sendo que cerca de $80 \%$ desse total foi recuperado nas lixiviações supercríticas, num tempo de 20 $\min$.
\end{abstract}

\section{INTRODUÇ̃̃̃O}

Na sociedade contemporânea, o consumo elevado, o ritmo acelerado da inovação e a chamada obsolescência programada fazem com que os equipamentos eletrônicos se transformem em sucata tecnológica em pouco tempo. São gerados, mundialmente, de 20 a 50 milhões de toneladas de resíduos de equipamentos elétricos e eletrônicos (EEE), o que representa um problema de ordem global(1,2). Estima-se que o Brasil tenha gerado aproximadamente 1,100 mil toneladas de resíduos de pequenos equipamentos eletroeletrônicos (REEE) em 2014, número este que deve aumentar para 1,247 mil toneladas em 2015 (3). Destacando-se os celulares, dentre esses pequenos EEEs.

As placas de circuito impresso (PCIs) são os componentes fundamentais dos EEE e motivam sua reciclagem, por serem compostas por uma quantidade significativa de metais (3). Segundo Yamane et al. (4), PCIs de celulares contém aproximadamente $63 \%$ de metais, $24 \%$ de cerâmicos e 13\% de polímeros. Os metais típicos presentes em PCIs são: $\mathrm{Cu}$ (presente em maior percentual), Fe, Sn, Ni, Pb, Zn, Ag, Au e Pd (Huang et al., 2009).

Os fluidos supercríticos também vêm sendo considerados como uma alternativa aos métodos convencionais (5). A utilização do dióxido de carbono $\left(\mathrm{CO}_{2}\right)$ vem sendo investigada, pois ele possui um ponto crítico relativamente baixo $\left(\mathrm{Tc}=31,1^{\circ} \mathrm{C}, \mathrm{Pc}=7,38 \mathrm{MPa}\right)$, é ambientalmente aceitável, pode ser reciclado e ser modificado com cosolventes (6). 
Além da necessidade da busca de métodos mais eficientes de reciclagem das PCIs, há o incentivo da Política Nacional de Resíduos Sólidos (PNRS), que foi instituída no Brasil em 2010 e estabelece a responsabilidade compartilhada na gestão dos resíduos sólidos. Diante deste contexto, este trabalho teve como objetivo desenvolver um método alternativo para recuperação do $\mathrm{Cu}$ de PCIs de celulares obsoletos empregando $\mathrm{CO}_{2}$ supercrítico e ácido sulfúrico $\left(\mathrm{H}_{2} \mathrm{SO}_{4}\right)$ e peróxido de hidrogênio $\left(\mathrm{H}_{2} \mathrm{O}_{2}\right)$ como cosolventes, avaliando a cinética reacional envolvida e o efeito do percentual de $\mathrm{H}_{2} \mathrm{O}_{2}$ utilizado na extração.

\section{MATERIAIS E MÉTODOS}

\subsection{Processamento Mecânico}

Por desmontagem manual, as PCIs foram retiradas de 29 celulares de mesma marca e modelo. Com um peso médio de 14,32g, essas placas forma cominuídas em um moinho de martelos (Tigre, modelo A4) com malha de $5 \mathrm{~mm}$. Seguidamente essa amostra foi cominuída novamente em um moinho de facas (Rone, modelo N-150) chegando-se a um diâmetro inferior a $2 \mathrm{~mm}$.

\subsection{CARACATERIZAÇÃO QUÍMICA DAS PCIs}

A determinação da composição química da amostra cominuída foi realizada por Espectrometria de Fluorescência de Raios-X com Energia Dispersiva (Shimadzu, modelo EDX720) à vácuo.

$\mathrm{O} \mathrm{Cu}$ presente nas PCIs foi quantificado a partir da lixiviação com água régia $\left(\mathrm{HNO}_{3}\right.$ e $\mathrm{HCl}$ na proporção de 1:3), utilizando $5 \mathrm{~g}$ de amostra cominuída, obtidos por quarteamento. Utilizou-se a razão sólido:líquido de $1 \mathrm{~g}: 50 \mathrm{~mL}$, a temperatura de $90^{\circ} \mathrm{C}$ e tempos de lixiviação de $1,2,3$ e 4 horas, sob agitação. Yamane et al. (2011) também utilizou água régia para quantificação dos metais existentes nas PCIs, mas em condições diferentes. As soluções lixiviadas foram separadas por filtração do material sólido e analisadas por Espectrometria de Absorção Atômica (Agilent Technologies, 200 Series AA). Para isso, estas foram diluídas até uma concentração máxima 10 $\mathrm{mg} / \mathrm{L}$ e acidificadas até $\mathrm{pH} 1,5$, garantindo-se assim que o $\mathrm{Cu}$ estivesse na forma solúvel (Welz e Spering, 1999). As diluições foram realizadas em duplicata e as análises em triplicata.

\subsection{Lixiviação Supercrítica}

A partir das PCIs cominuídas a um tamanho inferior a $2 \mathrm{~mm}$, obteve-se por quarteamento as amostras para serem utilizadas nos ensaios de lixiviação supercrítica. Essas amostras foram levadas ao sistema de extração supercrítico juntamente com os cosolventes.

O sistema utilizado nas extrações supercríticas é apresentado na Figura 1. As amostras e os cosolventes $\left(\mathrm{H}_{2} \mathrm{SO}_{4}\right.$ e $\left.\mathrm{H}_{2} \mathrm{O}_{2}\right)$ foram colocados, inicialmente, no reator de extração, que é constituído de aço inox 304, revestido internamente com teflon. O reator é encamisado, permitindo assim o controle de temperatura a partir de um banho ultratermostático com circulador (Quimis, modelo Q214M2). Depois da válvula de extração, além do frasco coletor, há outro frasco conectado contendo uma solução de hidróxido de sódio para realizar a lavagem do $\mathrm{CO}_{2}$ que se separa da solução extraída. $\mathrm{O} \mathrm{CO}_{2}(99,5 \%)$ é fornecido ao sistema já pressurizado e a pressão requerida é alcançada a partir de uma bomba de alta pressão do tipo seringa (Teledyne ISCO, modelo 500D).

Figura 1 - Sistema de extração supercrítica 

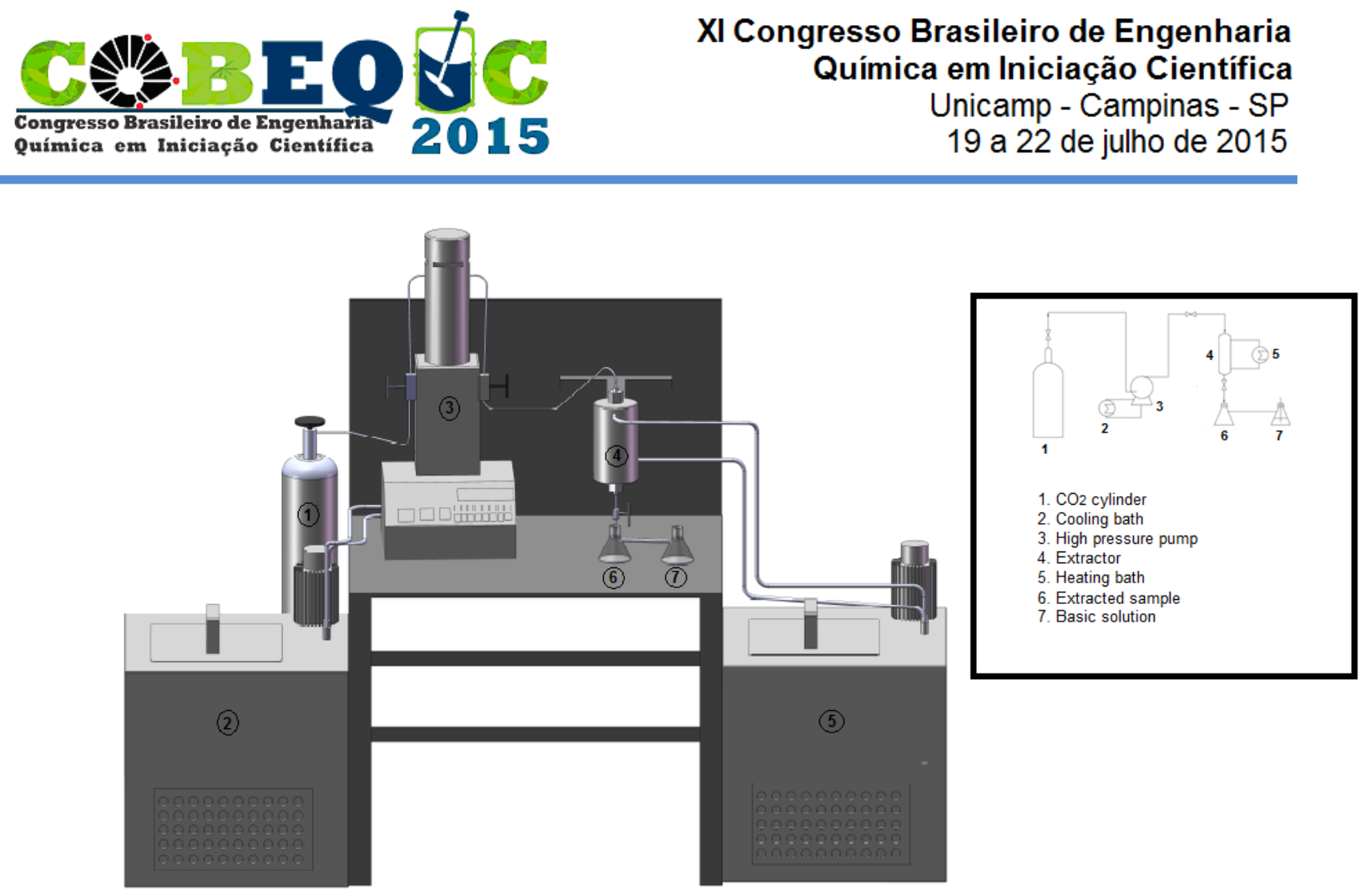

$\mathrm{O}$ efeito do percentual de $\mathrm{H}_{2} \mathrm{O}_{2}$, de 0 a $25 \%$, em relação à quantidade de $\mathrm{H}_{2} \mathrm{SO}_{4}$ como cosolvente, nas lixiviações supercríticas, foi avaliado no tempo ótimo determinado pelo estudo cinético, com as concentrações de $\mathrm{H}_{2} \mathrm{SO}_{4}$ de 2 e 2.5M, na razão sólido:líquido de 1:20.

O percentual mássico de $\mathrm{Cu}$ extraído foi determinado em relação ao percentual mássico de $\mathrm{Cu}$ lixiviado das amostras de placas cominuídas a partir da água régia, sendo este último considerado como valor total de $\mathrm{Cu}$ existente na amostra. A determinação da eficiência de recuperação é descrita pela Equação 1.

$\%$ Máximo de Cu recuperado $=\frac{\text { massa de Cu lixiviado X } 100}{\text { massa da amostra } \times \% \text { Cu recuperado com água régia }}$

\section{RESULTADOS}

\subsection{CARACATERIZAÇÃo QUÍMICA DAS PCIs}

A Tabela 1 apresenta a composição química das PCIs cominuídas, obtida por Espectrometria de Fluorescência de Raios-X com Energia Dispersiva (FRX). Onde se pode ver que o $\mathrm{Cu}$ é o metal presente em maior percentual mássico.

Tabela 1: Composição química das PCIs determinada por FRX. 


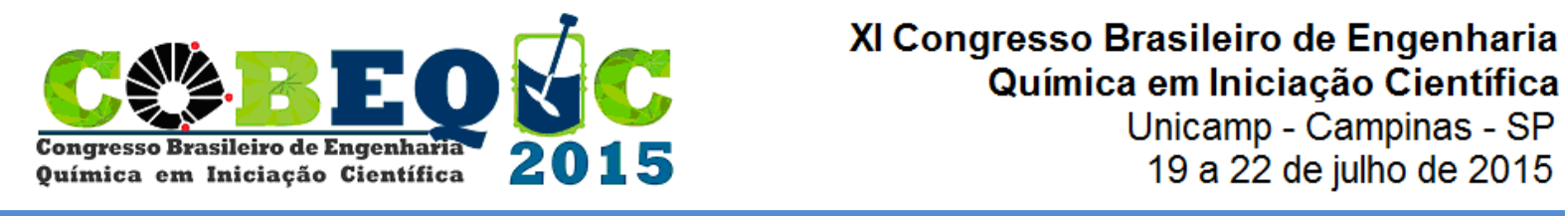

\begin{tabular}{cccccccccccccc}
\hline Elemento & $\mathrm{Cu}$ & $\mathrm{Si}$ & $\mathrm{Ca}$ & $\mathrm{Al}$ & $\mathrm{Ni}$ & $\mathrm{Ba}$ & $\mathrm{P}$ & $\mathrm{Fe}$ & $\mathrm{Sn}$ & $\mathrm{Ti}$ & $\mathrm{Zn}$ & $\mathrm{Ag}$ & Outros \\
\hline $\begin{array}{c}\text { Percentual } \\
\text { mássico } \\
(\%)\end{array}$ & 31.95 & 23.08 & 17.43 & 11.11 & 2.71 & 2.46 & 2.41 & 1.94 & 1.78 & 1.59 & 1.19 & 0.34 & 2.01 \\
$\mathbf{( \% )}$ & & & & & & & & & & & & & \\
\hline
\end{tabular}

A Figura 2 descreve a quantificação do $\mathrm{Cu}$ presente nas PCIs, empregando água régia como lixiviante. Pela Figura 3, observa-se que a variação do tempo não influenciou no percentual de $\mathrm{Cu}$ lixiviado com água régia, já que este foi semelhante nos tempos de 1, 2, 3 e 4 horas. O percentual mássico médio de $\mathrm{Cu}$ determinado a partir de lixiviação foi de $34,83 \%$, que se aproxima do valor obtido por FRX, de $31,95 \%$. Este valor é semelhante também ao determinado por Yamane et al. (2011), que foi de 34,5\% de $\mathrm{Cu}$ para PCIs de celulares. Assim, os 34,83\% de Cu resultante das lixiviações com água régia é considerado como a quantidade total de $\mathrm{Cu}$ existente nas PCIs de celulares estudadas.

Figura 2: Lixiviações com água-régia para quantificação do $\mathrm{Cu}$.

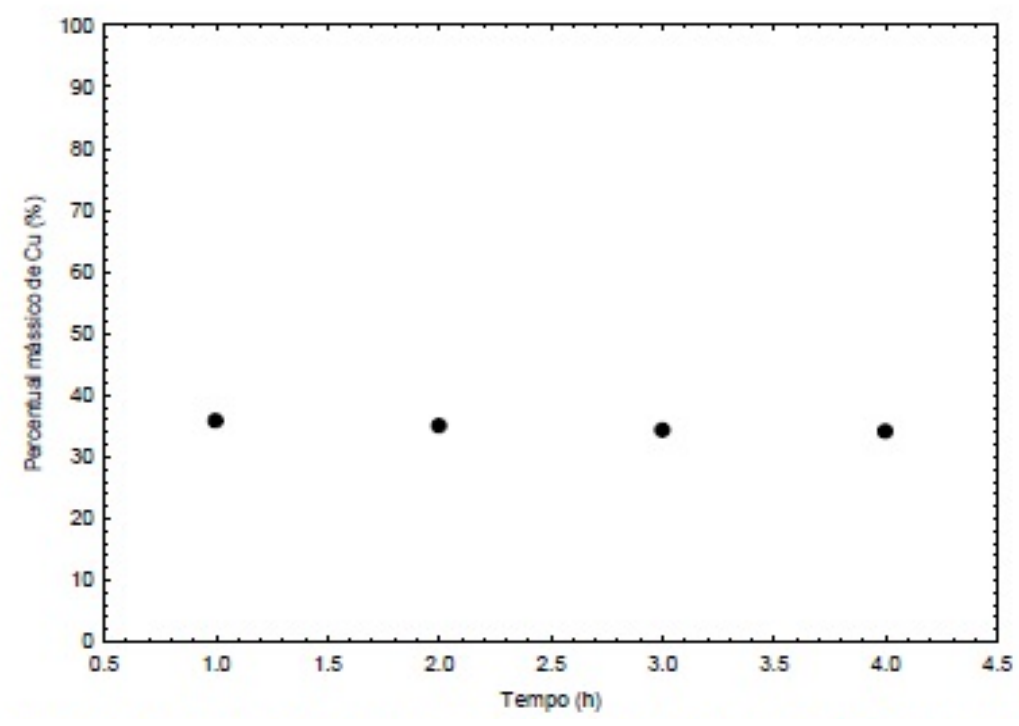

\subsection{LIXIVIAÇÕES}

Nas lixiviações ácidas com $\mathrm{CO}_{2}$ supercrítico (Figuras 3), lixiviou-se 51,7\% de $\mathrm{Cu}$ em apenas $1 \mathrm{~h}$ de reação e 48,5\% em 20 min. de reação (Figura 3), podendo-se dizer que em 20 min. a reação de lixiviação já se aproxima do equilíbrio.

Logo, com a lixiviação supercrítica se conseguiu um tempo reacional da lixiviação de 20 min. Entretanto, como nas lixiviações executadas se extraiu somente $50 \%$ do $\mathrm{Cu}$ presente, é necessário otimizar as condições operacionais, como a razão sólido-líquido ou até mesmo avaliar a possibilidade de se realizarem múltiplos estágios de extração para ter uma maior eficiência. 


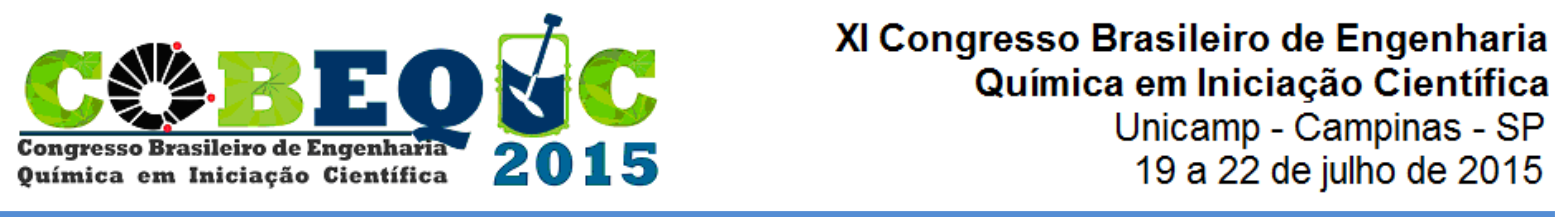

Figura 3: Cobre recuperado das PCIs de celular por extração supercrítica em diferentes razões sólido:líquido, com $\mathrm{H}_{2} \mathrm{SO}_{4}(2 \mathrm{M})$ e $15 \% \mathrm{H}_{2} \mathrm{O}_{2}(30 \%)$ cosolventes, em $35^{\circ} \mathrm{C}$ e $7.5 \mathrm{MPa}$.

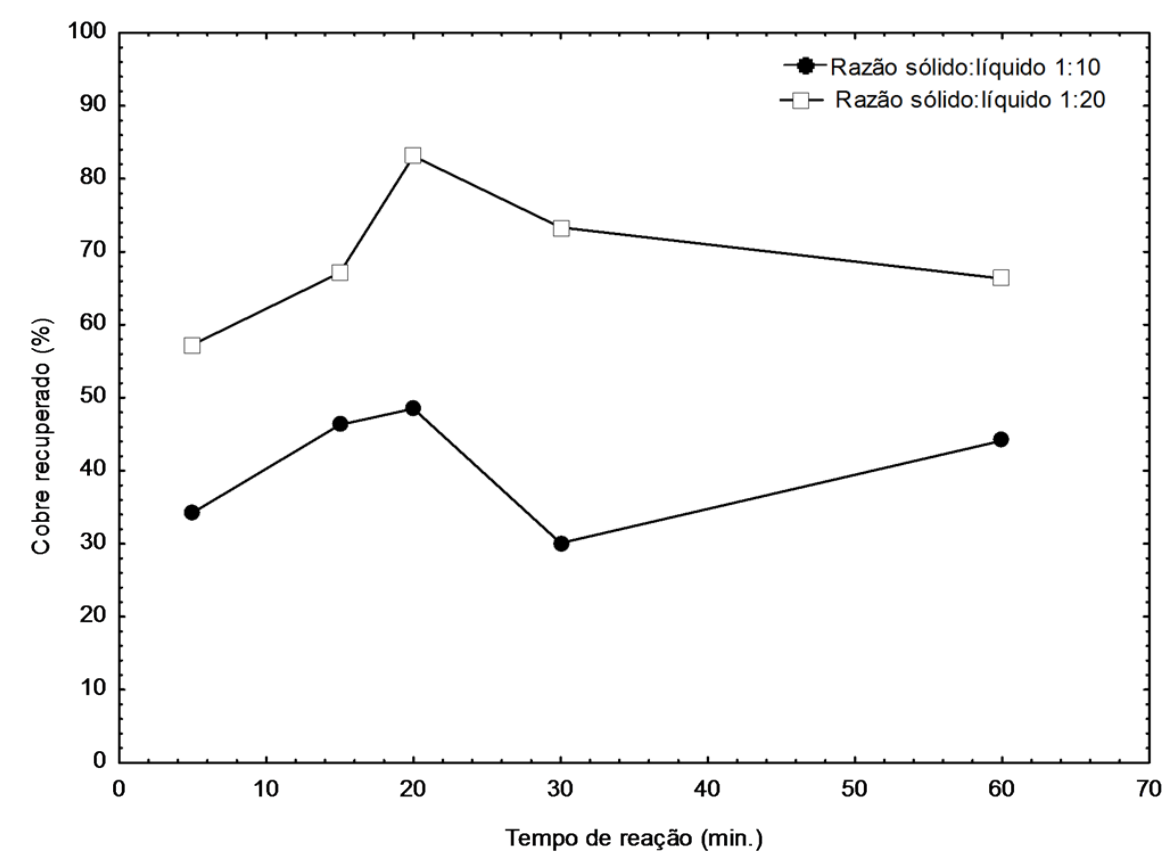

\subsection{EFEITOS DO PERCENTUAL DE $\mathrm{H}_{2} \mathrm{O}_{2}$ COMO COSOLVENTE}

$\mathrm{O}$ emprego do $\mathrm{H}_{2} \mathrm{O}_{2}$ como cosolvente juntamente com o $\mathrm{H}_{2} \mathrm{SO}_{4}$ nas lixiviações com $\mathrm{CO} 2$ supercrítico se mostrou determinante para recuperação do $\mathrm{Cu}$, pois sem ele o percentual de extração do metal foi inferior a $0,1 \%$, no tempo de $20 \mathrm{~min}$, para as concentrações de 2 e $2,5 \mathrm{M} \mathrm{de}_{2} \mathrm{SO}_{4}$.

$\mathrm{O}$ mesmo foi identificado por Yang et al. (2011), que recuperou menos de $5 \%$ do $\mathrm{Cu}$ contido em PCIs por lixiviação à pressão atmosférica, quando não foi adicionado $\mathrm{H}_{2} \mathrm{O}_{2}$. Podese verificar ainda, que o percentual de $20 \%$ de $\mathrm{H}_{2} \mathrm{O}_{2}$ da solução lixiviante de $2 \mathrm{M}$ e de $2,5 \mathrm{M}$ de $\mathrm{H} 2 \mathrm{SO} 4$ resultou nos maiores rendimentos de extração do $\mathrm{Cu}$, de $87,35 \%$ e de $88,79 \%$, respectivamente. Mas, quando o percentual de $\mathrm{H}_{2} \mathrm{O}_{2}$ aumenta para $25 \%$ e o de ácido se torna $75 \%$, a quantidade de $\mathrm{Cu}$ lixiviada se reduz novamente, indicando que depois do percentual de $20 \%$ de $\mathrm{H}_{2} \mathrm{O}_{2}$ em relação ao percentual de ácido, não é mais interessante aumentá-lo. Além disso, o aumento na concentração de ácido de 2 para 2,5 M não apresentou contribuição significativa para extração.

\section{CONCLUSÃO}

A caracterização química das PCIs a partir de FRX e lixiviações com água régia apresentou resultados próximos para a quantidade de $\mathrm{Cu}$ contido nas placas. Sendo o valor médio das lixiviações, de $34,83 \%$ adotado como referência, ou seja, como valor total de $\mathrm{Cu}$ presente nas 


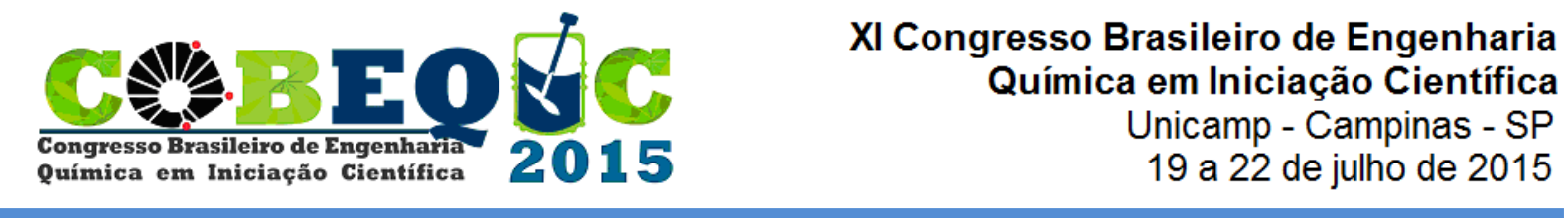

PCIs. Com as lixiviações empregando $\mathrm{CO}_{2}$ supercrítico recuperou-se $48,5 \%$ do total de $\mathrm{Cu}$ existente, porém com apenas $20 \mathrm{~min}$. de reação.

$\mathrm{O}$ emprego do $\mathrm{H}_{2} \mathrm{O}_{2}$ como cosolvente, juntamente com o ácido, foi significativo para a lixiviação supercrítica do $\mathrm{Cu}$. O percentual de $20 \%$ de $\mathrm{H}_{2} \mathrm{O}_{2}$ em relação ao ácido levou aos maiores percentuais de extração, recuperando-se $87,35 \%$ e $88,79 \%$ de $\mathrm{Cu}$ com $\mathrm{H}_{2} \mathrm{SO}_{4}$ nas concentrações de 2 e 2,5 M, respectivamente.

Portanto pode-se concluir que a lixiviação com $\mathrm{CO}_{2}$ supercrítico demonstrou um ganho de eficiência em termos de tempo, podendo ser uma alternativa promissora à reciclagem de PCIs e recuperação do $\mathrm{Cu}$ propriamente dita, mas carecendo ainda de estudos e de otimização das condições operacionais e das proporções dos reagentes.

\section{REFERÊNCIAS}

1. BEHNAMFARD, A.; SALARIRAD, M. M.; VEGLIO, F. Process Development for Recovery of Copper and Precious Metals from Waste Printed Circuit Boards with Emphasize on Palladium and Gold Leaching and Precipitation. Waste Manage., v. 33, p. 2354-2363, 2013.

2. BRASIL. Política Nacional de Resíduos Sólidos. Lei 12.305 de 2 de Agosto de 2010. 3. HERRERO, M.; MENDIOLA, J. A.; CIFUENTES, A.; IBÁÑEZ, E. Review: Supercritical fluid extraction: Recent advances and applications. J. Chromatogr. A, v. 1217, p. 2495-2511, 2010.

4. HUANG, K.; GUO, J.; XU, Z. Review - Recycling of waste printed circuit boards: A review of current technologies and treatment status in China. J. Hazard. Mater., v.164, p. 399-408, 2009.

5. LEE, C.; TANG, L.; POPURI, S. R. A study on the recycling of scrap integrated circuits by leaching. Waste Manage. Res., v. 29, p. 677-685, 2010.

6. LIN, F.; LIU, D.; MAITI, S.; PREMPEH, N.; HUA, Y.; LU, J. Recent Progress in Heavy Metal Extraction by Supercritical CO2 Fluids. Ind. Eng. Chem. Res., v. 53, p. 1866-1877, 2014.

PARK, Y.J.; FRAY, D.J. Recovery of high purity precious metals from printed circuit boards. J. Hazard. Mater., v. 164, p. 1152-1158, 2009. 